\section{Morbidity Patterns Amongst Hospitalized Patients in a Secondary Care Hospital of Dehradun, Uttarakhand State, India}

\section{Brij Bhushan Tyagi ${ }^{1 *}$, Aswal RS $^{2}$ and Haroon AS $^{3}$}

${ }^{1}$ Department of Medical Health and Family Welfare, National Rural Health Mission, Government of Uttarakhand, Uttarakhand, Dehradun, India

\section{${ }^{2}$ Government Doon Hospital, Dehradun, Uttarakhand, India}

${ }^{3}$ Department of Community Medicine, Veer Chandra Singh Garhwali Government Institute of Medical Science and Research, Srinagar, Pauri Garhwal, Uttarakhand, India

\begin{abstract}
Introduction: In India, there is a paucity of morbidity and mortality information de-segregated at facility level. Present study was undertaken to report the morbidity and mortality of in-patient at a secondary care hospital. There are no data available on morbidity and mortality patterns in hospitalized patients of the state of Uttarakhand in India.

Materials and methods: Study was conducted in a secondary care Government Doon Hospital at Dehradun, Uttarakhand State, India. It is a record based retrospective study. Diagnoses are coded as per the International statistical Classification of Diseases (ICD.10). Relevant information pertaining to socio-demographic indicators, diagnosis and outcome at discharge about inpatients (1/1/2012 to $31 / 12 / 2012$ ) were retrieved from HMIS archives. Descriptive analysis was done.

Results: During the study period, a total of 18309 patients were admitted in all departments/wards. Out of these 10735 (58.6\%) were males and $7574(41.4 \%)$ were females. A statistically significant difference was seen between males and females for morbidity disease category of eye and adnexa [ICD.10: H00-H59, 3.3 ( $p<0.05)$ ] with subcategory of disease [ICD.10:H25.2, $2.5(p<0.05)]$, diseases of the respiratory system [ICD.10:J00-99, $2.2(p<0.05)]$ and morbidity disease category of injury, poisoning and certain other consequences of external causes [ICD.10:S00-T98, $3.0(p<0.05)]$. The average bed
\end{abstract}

*Corresponding author: Brij Bhushan Tyagi, Department of Medical Health and Family Welfare, National Rural Health Mission, Government of Uttarakhand, Uttarakhand, Dehradun, India, Tel: +91 9560026481; E-mail: btyagi51@gmail.com

Citation: Tyagi BB, Aswal RS, Haroon AS (2015) Morbidity Patterns Amongst Hospitalized Patients in a Secondary Care Hospital of Dehradun, Uttarakhand State, India. J intern Med Prim Healthcare 1: 003.

Received: May 18, 2015; Accepted: July 20, 2015; Published: August 03, 2015 occupancy rate of the hospital was 97.2 percent recorded throughout the year and overall mortality rate was $40.4 / 1000$ admissions.

Conclusion: The present study only highlights the status of different types of morbidity, mortality and bed occupancy rates in a secondary care hospital. This is a record based study.

Key message: There is need to upgrade existing health infrastructure at secondary care level facility to increase bed occupancy and minimize referral rate. The reasons for the high LAMA rate needs to be studied in detail to bring it down.

Keywords: Dehradun; In-patients; Secondary care hospital

\section{Introduction}

India is a large country with huge variations in health indicators across states and districts of the country [1]. Unlike other big nations, for instance Russia, China and the USA, the Indian population consists of many communities with different cultures and habits. The morbidity and mortality pattern across India varies significantly as would be expected from the wide health and health determinant disparity spread across length and breadth of India $[2,3]$.

Life expectancy at birth, mortality and morbidity rates are important indicators of health status of a population. In India the life expectancy has increased from 23 years in 1901 to 62.6 in 2002-06, and infant mortality has declined from 215 per thousand in 1901 to 50 in 2009. The death rates have declined from 44 per 1000 in 1901 to 10 in 1992 and 7.3 in 2009 [4]. However, there are no realistic and comparable estimates of morbidity over a period of time. The life expectancy at birth is an important indicator of quality of the people. The estimates of morbidity in general and the disease specific incidence rates in particular would serve as valuable information to the health planners and administrators for appropriate and timely measures to monitor, control and eradicate the diseases. It will also enable the administrators to allocate resources for health facilities such as hospitals, physicians, medicines, etc., and provide basic infrastructure such as sanitation and drinking water. Recently morbidity estimates have been used to assess the burden of diseases [5]. Although a low morbidity rate can be considered as an indicator of better health status, it need not necessarily be true. Low morbidity can occur from actual reduction in incidence of illness or due to under reporting or due to lack of disease consciousness. There is considerable disagreement among researchers on how to define and measure morbidity since it is highly subjective based on perception and reporting. In certain cases morbidity may be the reflection of poverty itself [6]. In India, there is a paucity of morbidity and mortality information de-segregated at facility level. There have been few studies documenting the morbidity profile of a population over a period of time in other parts of India, but none in the state of Uttarakhand [7]. Central Bureau of Health Information (CBHI) does report aggregated state level and national level morbidity information but this information has limited use at district and sub-district level [1].

National Rural Health Mission (NRHM) has emphasized the importance of de-centralization of health system policy and planning. Following this, there is renewed interest in health planning at district 
and sub-district level. One of the prerequisites of decentralized planning is the availability of locally relevant valid information including morbidity and mortality statistics to guide planning $[8,9]$.

The overload in hospital ward remains a major source of concern in many countries, including India, for policy makers. The availability of hospital beds has always been a problem in developing countries. It is the most important single factor in determination of the utilization of hospital beds in a country [10]. In India, shortage of hospital beds is a huge problem, the average bed population ratio being 9 per 10,000 population in comparison with the world average of 27 per 10,000 during 2000-2009 [11].

Morbidity and mortality reporting of in-patients will not only provide valuable information regarding burden and cause of illness but also enable us to assess the adequacy of the available facilities. It will further provide inputs to restructure and upgrade the existing health services. Present study was undertaken to find out the pattern and distribution of morbidities, case fatality rate and bed occupancy rate of in-patient at a secondary care hospital at Dehradun with aim to identify key areas that require improvement in the existing health system and also for planning and resource allocation in a similar secondary level health settings. To our knowledge, there has not been any study documenting the morbidity profile of admissions in a secondary care hospital of Uttarakhand State, which will aid in deciding whether specialists staffing is required or not. We therefore felt the need to record the morbidity profile of admissions in a secondary healthcare hospital. The hospital patients consist of private fee-paying patients and staff/dependents of government or private companies, which have retained the services of the hospital. The patients therefore represent various strata of the society cutting across social classes, religions and ethnic groups. The place of residence of majority of the patients extended from close vicinity of the hospital to a distance as far as $100 \mathrm{~km}$ approximately. The worst affected were villagers who trekked several kilometers from the hilly terrains of the State. In view of the increasing patient load, the Uttarakhand State Government has taken the decision to convert the "Doon Hospital" to a medical college [12].

\section{Material and Methods}

\section{Study population and study period}

The secondary care Doon Hospital caters primarily to the population of Dehradun districts which includes urban, slum and rural areas. As per census 2011 report, the total population of Dehradun district was around 1,698,560 [males - 893,222 (52.6\%), females - 805,338 (47.4\%)] [13]. All the patients admitted at hospital from $1^{\text {st }}$ January, 2012 to $31^{\text {st }}$ December, 2012 were included for the morbidity and mortality analysis.

\section{Study design and study settings}

This was a retrospective (record based) review study. The hospital has more than 40 allopathic doctors including pediatricians, physicians, surgeons, neurophysicians, orthosurgeons, Ear-Nose-Throat (ENT) specialists and eye surgeons besides doctors belonging to AYUSH (Ayurveda, Yoga, Naturopathy, Unani, Siddha and Homoeopathy) providing medical aid. The patients are admitted under clinical disciplines of pediatrics, internal medicine, surgery, neurology, cardiac, skin, ENT, dental and ophthalmology. The admissions were done under either routine or emergency category. The discharge of a patient was based on individualized assessment and clinical outcome of the treatment administered. The hospital followed the following categories of condition at discharge: 1 . Cured; 2. Relieved; 3. Referred; 4. LAMA and 5. Expired. Obstetrics and gynecology facilities are not available in Doon Hospital. There is a separate female hospital adjacent to the hospital premises for obstetrics and gynecology and other diseases. Seriously ill patients requiring higher level of care are referred to other government-funded hospitals in the district. Ambulance facilities are available at the hospital round the clock for patients' use.

\section{Hospital Management Information System (HMIS)}

HMIS started functioning in the hospital from 2008 under the National Rural Health Mission. All information pertaining to out-door and in-patients are entered and stored in HMIS.

\section{Data retrieval}

The details of patients and their diagnosis were collected from the case sheets of the patients or discharge registers kept in medical records department. Relevant information pertaining to socio-demographic indicators, diagnosis, treatment, prognosis of the disease condition at discharge and cause of death etc., as recorded in the case records by the clinicians from period of $1^{\text {st }}$ January, 2012 to $31^{\text {st }}$ December, 2012 was retrieved from HMIS archives. Records of patients with missing information on bio-data and diagnosis were excluded from study. For those with multiple diagnoses, morbidity with the longest duration or the final diagnosis (supported by relevant laboratory investigations) was recorded as primary illness for the patient. All the information collected was cross-checked for completeness of the data from the records available at hospital (viz- patient's admission file, reports and ward registers, etc.). The World Health Organization (WHO) standards were used for analyzing and comparing the data. The abstracted data is coded by using manual of International Statistical Classification of Disease and Related Health Problems (10 $0^{\text {th }}$ revision), Volume $1 \& 2$, Second Edition published by the WHO, Geneva [14].

\section{Permission}

Specifying that written permission for conducting the study was obtained prior to collecting the data from the additional health secretary and mission director, NRHM, Director General of Health Services (DGHS) of the Uttarakhand state and also from medical superintendent of the Doon Hospital by explaining the purpose of the study. Strict confidentiality was maintained on the data collected. No financial sources were involved to conduct this study also no ethical clearance was required to conduct this study.

\section{Statistical Analysis}

Collected data were entered in Microsoft Excel and were analyzed using software Statistical Package for Social Sciences (SPSS) version 21.0. Descriptive statistical measures such as percentage, mean were applied. Inferential statistical tests such as Z-test and Chi-square test were applied to identify important relationships between variables and determine the level of significance. A p-value of $<0.05$ was considered statistically significant.

\section{Results}

Table 1 shows distribution of patients according to morbidity, mortality and Case Fatality Rate (CFR) by 5 year age groups and gender. A total of 18,309 patients were admitted in all the wards from $1^{\text {st }}$ January, 2012 to $31^{\text {st }}$ December, 2012. Of these 10,735 (58.6\%) were 
Citation: Tyagi BB, Aswal RS, Haroon AS (2015) Morbidity Patterns Amongst Hospitalized Patients in a Secondary Care Hospital of Dehradun, Uttarakhand State, India. J intern Med Prim Healthcare 1: 003.

- Page 3 of $10 \bullet$

\begin{tabular}{|c|c|c|c|c|c|c|c|c|c|c|c|c|c|c|c|}
\hline \multirow{3}{*}{ Ages } & \multicolumn{5}{|c|}{ Male } & \multicolumn{5}{|c|}{ Female } & \multicolumn{5}{|c|}{ Total } \\
\hline & \multicolumn{2}{|c|}{ Morbidity } & \multicolumn{2}{|c|}{ Mortality } & \multirow{2}{*}{ CFR } & \multicolumn{2}{|c|}{ Morbidity } & \multicolumn{2}{|c|}{ Mortality } & \multirow{2}{*}{ CFR } & \multicolumn{2}{|c|}{ Morbidity } & \multicolumn{2}{|c|}{ Mortality } & \multirow{2}{*}{ CFR } \\
\hline & No. & $\%$ & No. & $\%$ & & No. & $\%$ & No. & $\%$ & & No. & $\%$ & No. & $\%$ & \\
\hline $1-4$ & 681 & 3.8 & 3 & 0.4 & 0.4 & 380 & 2.1 & 7 & 0.9 & 1.8 & 1061 & 5.8 & 10 & 1.3 & 0.9 \\
\hline $5-9$ & 552 & 3.0 & 5 & 0.7 & 0.9 & 379 & 2.1 & 5 & 0.7 & 1.3 & 931 & 5.1 & 1 & 0.1 & 1.1 \\
\hline $10-14$ & 518 & 2.9 & 6 & 0.8 & 1.2 & 388 & 2.1 & 3 & 0.4 & 0.8 & 906 & 4.9 & 9 & 1.2 & 1.0 \\
\hline $15-19$ & 679 & 3.7 & 9 & 1.2 & 1.3 & $569^{2}$ & 3.1 & 8 & 1.1 & 1.4 & $1248^{2}$ & 6.8 & 17 & 2.3 & 1.4 \\
\hline $20-24$ & $987^{1}$ & 5.4 & 14 & 1.9 & 1.4 & $604^{1}$ & 3.3 & 16 & 2.2 & 2.6 & $1591^{1}$ & 8.7 & 30 & 4.0 & 1.9 \\
\hline $25-29$ & $743^{3}$ & 4.1 & 27 & 3.6 & 3.6 & 457 & 2.5 & 6 & 0.8 & 1.3 & $1200^{5}$ & 6.5 & 33 & 4.5 & 2.8 \\
\hline $30-34$ & 624 & 3.4 & 28 & 3.8 & 4.5 & 461 & 2.5 & 13 & 1.8 & 2.8 & 1085 & 5.9 & 41 & 5.5 & 3.8 \\
\hline $35-39$ & 675 & 3.7 & $42^{5}$ & 5.7 & 6.2 & $485^{5}$ & 2.6 & 13 & 1.8 & 2.7 & 1160 & 6.3 & 55 & 7.4 & 4.7 \\
\hline $40-44$ & 681 & 3.7 & 31 & 0.1 & 4.6 & $489^{4}$ & 2.7 & 18 & 2.4 & 3.7 & 1170 & 6.4 & 49 & 6.6 & 4.2 \\
\hline $50-54$ & $761^{2}$ & 4.2 & $48^{3}$ & 6.5 & 6.3 & 453 & 2.5 & 13 & 1.8 & 2.9 & $1214^{4}$ & 6.6 & $61^{3}$ & 8.2 & 5.0 \\
\hline $55-59$ & 578 & 3.2 & 37 & 5.0 & $6.4^{5}$ & 427 & 2.3 & $21^{5}$ & 2.8 & $4.9^{4}$ & 1005 & 5.5 & $58^{5}$ & 7.8 & $5.8^{5}$ \\
\hline $60-64$ & $684^{5}$ & 3.7 & $60^{1}$ & 8.1 & $8.8^{1}$ & $560^{3}$ & 3.1 & $27^{2}$ & 3.6 & $4.8^{5}$ & $1244^{3}$ & 6.8 & $87^{2}$ & 11.8 & $7.0^{2}$ \\
\hline $65-69$ & 504 & 2.7 & 37 & 5.0 & $7.3^{3}$ & 435 & 2.4 & $23^{4}$ & 3.1 & $5.3^{3}$ & 939 & 5.1 & $60^{4}$ & 8.1 & $6.4^{4}$ \\
\hline $70-74$ & 499 & 2.7 & 36 & 4.9 & $7.2^{4}$ & 390 & 2.1 & $24^{3}$ & 3.2 & $6.2^{2}$ & 889 & 4.9 & $60^{4}$ & 8.1 & $6.7^{3}$ \\
\hline $75+$ & 565 & 3.1 & $49^{2}$ & 6.6 & $8.7^{2}$ & 417 & 2.3 & $41^{1}$ & 5.5 & $9.8^{1}$ & 982 & 5.4 & $90^{1}$ & 12.2 & $9.2^{1}$ \\
\hline Total & 10735 & 58.6 & 482 & 65.1 & 4.5 & 7574 & 41.4 & 258 & 34.9 & 3.4 & 18309 & 100.0 & 740 & 100.0 & 4.0 \\
\hline \multicolumn{3}{|c|}{ Z- Statistics } & \multicolumn{8}{|c|}{ Morbidity $=22.9(p<.001)^{\star *}$} & \multicolumn{5}{|c|}{ Mortality $=7.9(p<.001)^{\star *}$} \\
\hline
\end{tabular}

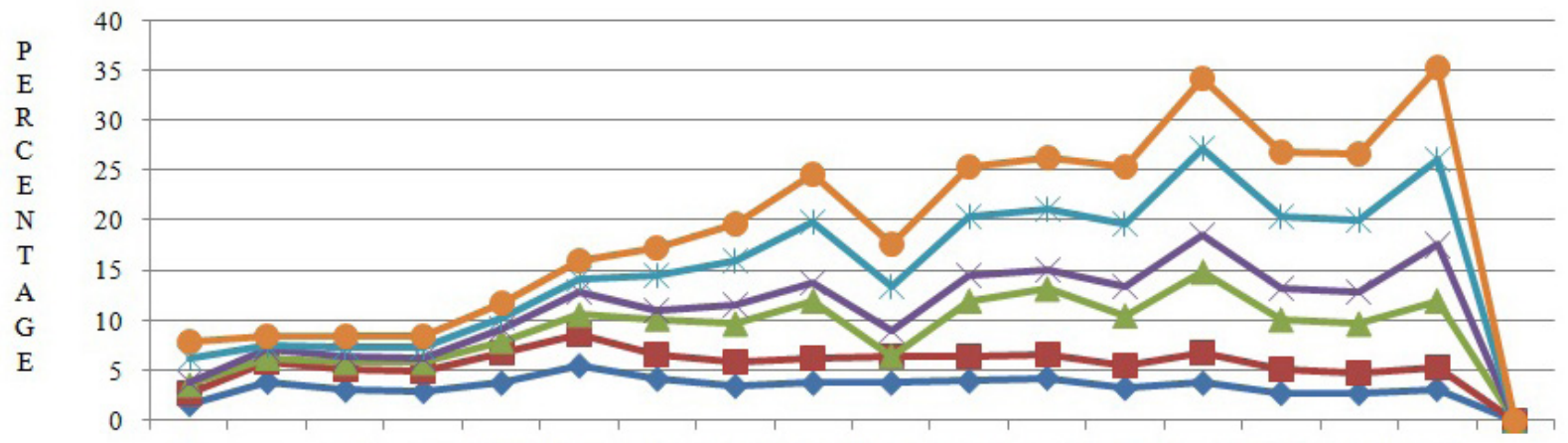

$\begin{array}{lllllllllllllllll}0-1 & 1-4 & 5-9 & 10-14 & 15-19 & 20-24 & 25-29 & 30-34 & 35-39 & 40-44 & 45-49 & 50-54 & 55-59 & 60-64 & 65-69 & 70-74 & 75+\end{array}$

Age groups

$\longrightarrow$ Male Morb $\rightarrow-$ Female Morb $\longrightarrow$ Male Mort $\longrightarrow$ Female Mort $\longrightarrow$ Male CFR $\multimap$ Female CFR

Figure 1: Distribution of patients according to morbidity, mortality and Case Fatality Rate (CFR) by age and gender.

males and 7,574 (41.4\%) were females resulting in a sex ratio of males 1000 to females 705 . The highest number of admissions came from the 20-24 year old age group in both sexes [male $\mathrm{n}=987(5.4 \%)$, females $\mathrm{n}=604(3.3 \%)]$ as compared to other age groups. In males the highest number of deaths/mortality belonged to the 60-64 year old age group [n=60 (8.1\%)] followed by the $75+$ years old age group $[n=49(6.6 \%)]$ while in females the highest number of deaths/mortality belonged to the $75+$ year old age group $[n=41(5.5 \%)]$ followed by the $60-64$ year old age group $[n=27(3.6 \%)]$. Over all CFR were 4.0 per 1000 for both sexes (male $-4.5 \%$, female $-3.4 \%$ ) (Figure 1 ), statistically significant 
Citation: Tyagi BB, Aswal RS, Haroon AS (2015) Morbidity Patterns Amongst Hospitalized Patients in a Secondary Care Hospital of Dehradun, Uttarakhand State, India. J intern Med Prim Healthcare 1: 003.

- Page 4 of $10 \cdot$

\begin{tabular}{|c|c|c|c|c|c|c|c|c|c|c|c|c|c|}
\hline \multirow{2}{*}{ Age group } & \multicolumn{2}{|c|}{ Cured } & \multicolumn{2}{|c|}{ Relieved } & \multicolumn{2}{|c|}{ Referred } & \multicolumn{2}{|c|}{ LAMA } & \multicolumn{2}{|c|}{ Expired } & \multicolumn{2}{|c|}{ Total } & \multirow{2}{*}{ Chi square } \\
\hline & No. & $\%$ & No. & $\%$ & No. & $\%$ & No. & $\%$ & No. & $\%$ & No. & $\%$ & \\
\hline $0-14$ & 2219 & 21.6 & 710 & 19.9 & 80 & 12.9 & 342 & 11.0 & 38 & 5.1 & 3389 & 18.5 & $234.28(p<.001)^{\star \star}$ \\
\hline $15-34$ & 2688 & 26.2 & 871 & 24.4 & 171 & 27.6 & 1273 & 40.9 & 121 & 16.4 & 5124 & 28.0 & $249.6(p<.001)^{* *}$ \\
\hline $35-64$ & 3729 & 36.3 & 1466 & 41.2 & 286 & 46.2 & 1132 & 36.4 & 371 & 50.1 & 6986 & 38.2 & $58.94(\mathrm{p}<.001)^{* *}$ \\
\hline $65+$ & 1635 & 15.9 & 517 & 14.5 & 82 & 13.2 & 366 & 11.8 & 210 & 28.4 & 2810 & 15.3 & $112.71(p<.001)^{\star \star}$ \\
\hline Total & 10271 & 100.0 & 3566 & 100.0 & 619 & 100.0 & 3113 & 100.0 & 740 & 100.0 & 18309 & 100.0 & \\
\hline$\%$ & \multicolumn{2}{|c|}{$56.1^{1}$} & \multicolumn{2}{|c|}{$19.5^{2}$} & \multicolumn{2}{|c|}{$3.4^{5}$} & \multicolumn{2}{|c|}{$17.0^{3}$} & \multicolumn{2}{|c|}{$4.0^{4}$} & \multicolumn{2}{|c|}{100.0} & \\
\hline
\end{tabular}

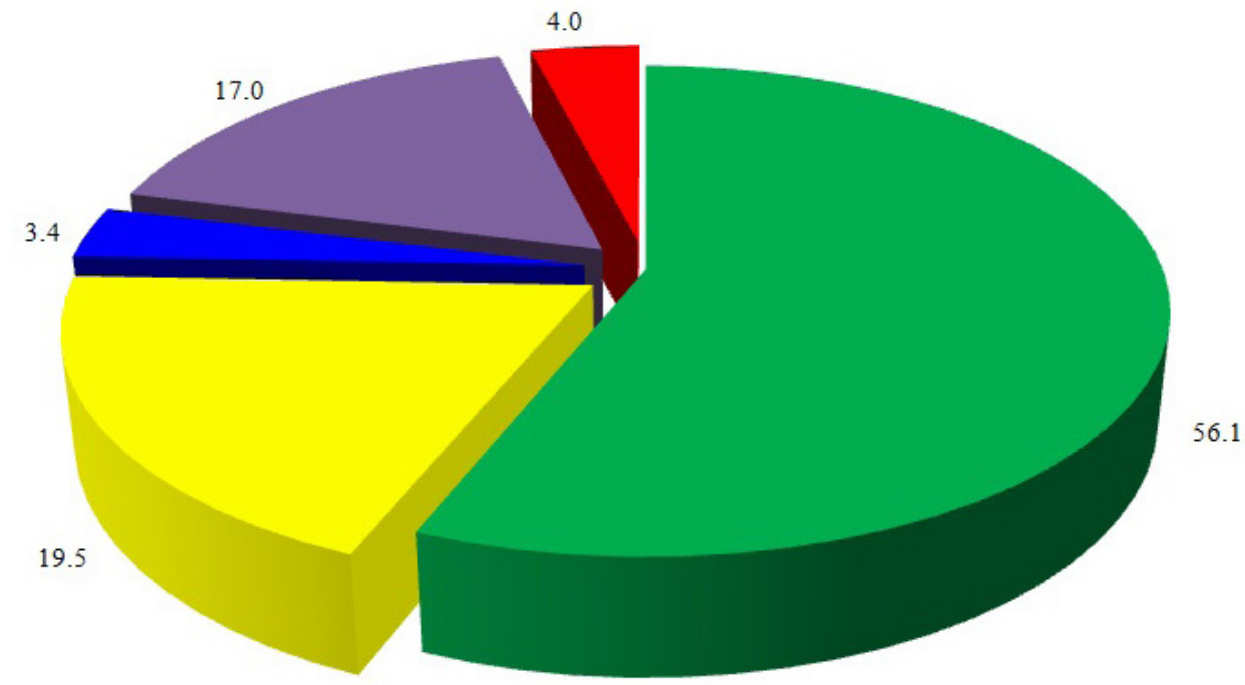

- Cured $\quad$ Relieved $\quad$ Referred $\quad$ LAMA Expired

Figure 2: Percentage distribution of patients according to condition at discharge.

association was found between age groups, morbidity $(22.9, \mathrm{p}<0.001)$ and in mortality $(7.9, \mathrm{p}<0.001)$ by sex.

Table 2 shows the distribution of patients according to condition at discharge and ages. It was found that out of the total $(n=18309)$ admitted cases in hospital among various wards, 10271 patients (56.1\%) were discharged as cured, 3566 (19.5\%) were relieved, 3113 (17.0\%) Left Against Medical Advice (LAMA), 740 (4.0\%) patients expired and the remaining 619 (3.4\%) were referred (Figure 2). Statistically significant association was found between the age groups and patients discharge conditions.

Dehradun, the capital city of the state of Uttarakhand, is one of the most visited tourist destinations in India that attracts both domestic and international visitors. The climate of Dehradun is generally temperate, although it varies from hot in summers (up to $40^{\circ} \mathrm{C}$ ) to severely cold (down to $1^{\circ} \mathrm{C}$ ) in winters. Although the nearby hilly regions often get snowfall during winter, in Dehradun it is not a frequent occurrence. During the monsoon season, there is often heavy and protracted rainfall. Dehradun and other plains areas of Uttarakhand state see almost as much rainfall as coastal areas of Maharashtra state and more than the state of Assam [15].
Table 3 provides the distribution of seasonal variations in morbidity, mortality, CFR and Bed Occupancy Rate (BOR). The climate in Dehradun area cannot be exactly differentiated into the conventional four seasons. A more practical demarcation is the winter months of December, January and February, the spring season months of March and April, the hot-dry period of May and June, the hot-wet monsoon (rainy) period of July, August and September, and autumn comprising October and November. Out of the total admission ( $\mathrm{n}=18309)$, the high point in the frequency of morbidities [both sexes: $\mathrm{n}=4696$ (25.6\%), male: 2785 and female: 1911] and mortalities [ $\mathrm{n}=181$ (24.5\%), male: 118 and female: 63] were found in the hot-wet season (July to September month). Overall CFR was 4.0 per 1000 population (male: 4.5 and female: 3.4 ). A high (4.7) case fatality rate per 1000 population was seen in the autumn season (October: 5.3, November: 3.9) and minimum (3.0) in winter seasons (December: 2.9, January: 3.6 and February: 2.6). Overall BOR was 97.2 percent. The month of May saw very high BOR (113.8\%) followed by July (103.6), August (102.7) and September (102.3). January recorded the lowest BOR (81.8) (Figure 3). A z-test was applied to know the statistical significance difference among the BOR during different seasons of the year. Statistically highly significant 
Citation: Tyagi BB, Aswal RS, Haroon AS (2015) Morbidity Patterns Amongst Hospitalized Patients in a Secondary Care Hospital of Dehradun, Uttarakhand State, India. J intern Med Prim Healthcare 1: 003.

- Page 5 of $10 \bullet$

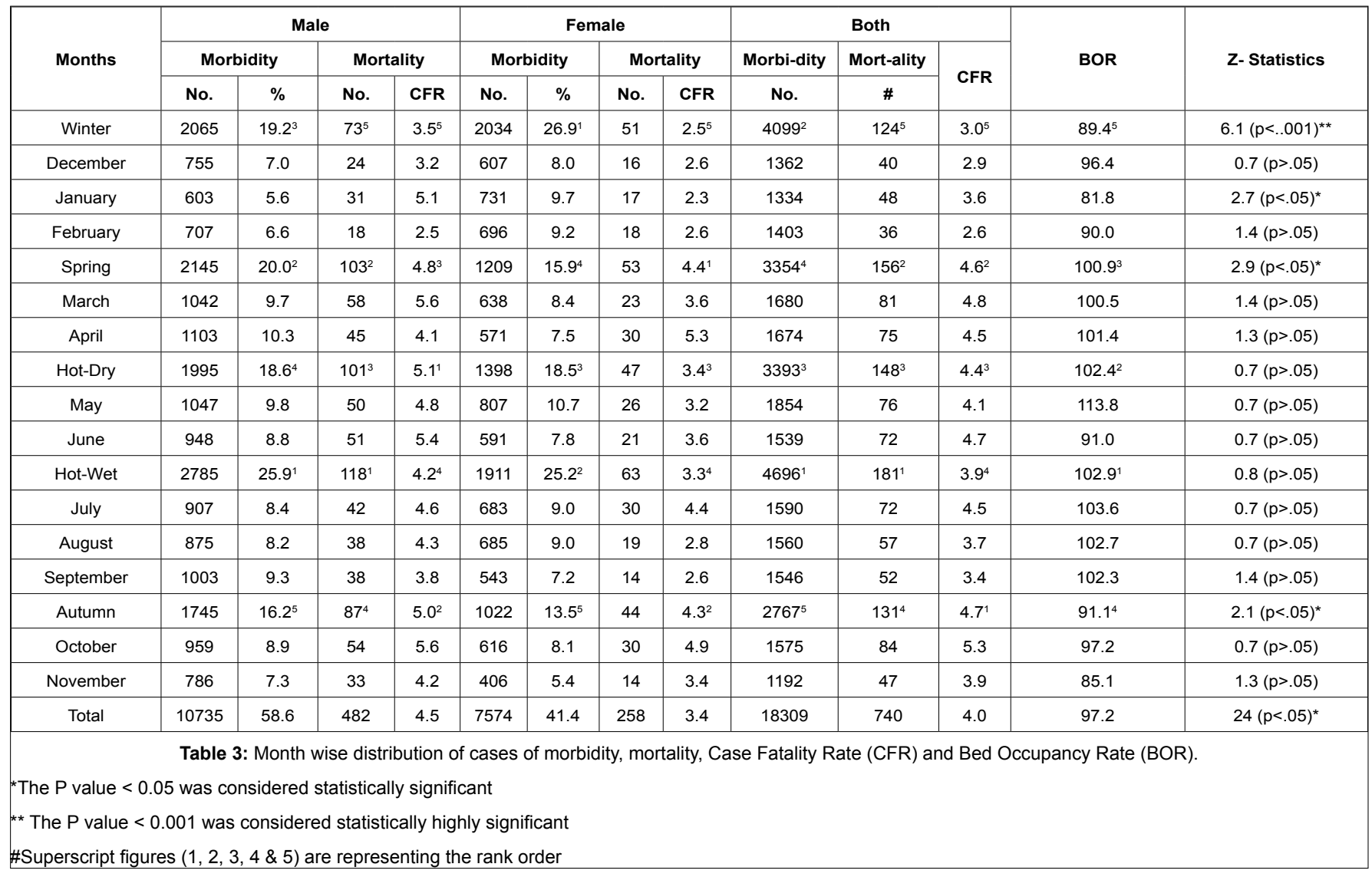

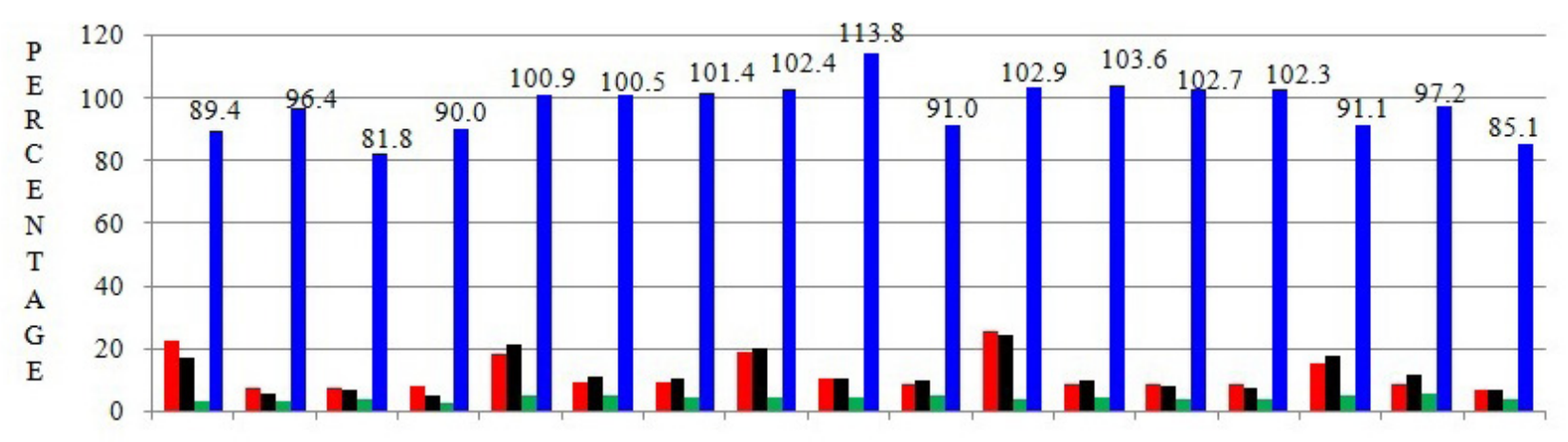

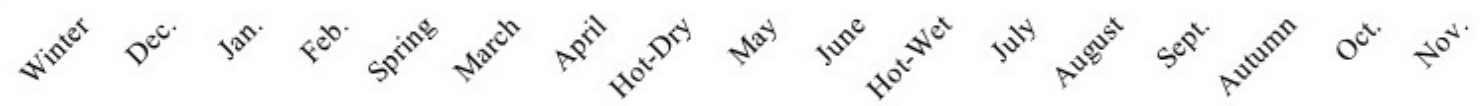

MONTHS

- Morbidity Mortality $\quad$ Case Fatality Rate Bed Occupancy Rate

Figure 3: Month wise distribution of patient admitted, death cases, Case Fatality Rate (CFR) and Bed Occupancy Rate (BOR), Total Cases ( $n=18309$ ).

difference was observed during the winter season $(6.1, \mathrm{p}<0.001)$ also statistically significant difference was observed in spring season $(2.9, \mathrm{p}<0.05)$ and in autumn season $(2.1, \mathrm{p}<.05)$.
Table 4 depicts the morbidity and mortality pattern of diseases among patients admitted in this secondary care hospital. The leading causes of morbidity in males were symptoms, signs and 
Citation: Tyagi BB, Aswal RS, Haroon AS (2015) Morbidity Patterns Amongst Hospitalized Patients in a Secondary Care Hospital of Dehradun, Uttarakhand State, India. J intern Med Prim Healthcare 1: 003.

- Page 6 of 10 -

\begin{tabular}{|c|c|c|c|c|c|c|c|c|c|c|c|c|c|c|c|}
\hline \multirow{3}{*}{$\begin{array}{l}\bar{\gamma} \\
\stackrel{0}{0} \\
\varnothing \\
\circ \\
\circ\end{array}$} & \multirow{3}{*}{$\begin{array}{l}\text { Disease } \\
\text { Category } \\
\text { group }\end{array}$} & \multirow{3}{*}{$\begin{array}{c}\text { Leading } \\
\text { Sub-category of } \\
\text { disease (ICD.10) }\end{array}$} & \multicolumn{4}{|c|}{ Male } & \multicolumn{4}{|c|}{ Female } & \multicolumn{4}{|c|}{ Total } & \multirow{3}{*}{$\begin{array}{c}\text { Z Statistics with } \\
\text { p-value }\end{array}$} \\
\hline & & & \multicolumn{2}{|c|}{ Morbidity } & \multicolumn{2}{|c|}{ Mortality } & \multicolumn{2}{|c|}{ Morbidity } & \multicolumn{2}{|c|}{ Mortality } & \multicolumn{2}{|c|}{ Morbidity } & \multicolumn{2}{|c|}{ Mortality } & \\
\hline & & & No. & $\%$ & No. & $\%$ & No. & $\%$ & No. & $\%$ & No. & $\%$ & No. & $\%$ & \\
\hline \multirow{4}{*}{ (A00-B99) } & \multicolumn{2}{|c|}{$\begin{array}{l}\text { Certain infectious } \\
\text { and parasitic diseases }\end{array}$} & 1799 & 16.76 & 62 & 12.86 & 1332 & 17.59 & 34 & 13.18 & 3131 & 17.10 & 96 & 12.97 & $0.6(p>.05)$ \\
\hline & & A09 & 1002 & 9.33 & 6 & 1.24 & 858 & 11.33 & 18 & 6.98 & 1860 & 10.16 & 24 & 3.24 & $0.5(p>.05)$ \\
\hline & & A01.0 & 240 & 2.24 & 0 & 0.00 & 225 & 2.97 & 0 & 0.00 & 465 & 2.54 & 0 & 0.00 & $1.4(p>.05)$ \\
\hline & & A16.9 & 256 & 2.38 & 23 & 4.77 & 99 & 1.31 & 8 & 3.10 & 355 & 1.94 & 31 & 4.19 & $0.1(p>.05)$ \\
\hline \multirow{4}{*}{ (C00-D48) } & \multicolumn{2}{|c|}{ Neoplasms } & 44 & 0.41 & 3 & 0.62 & 82 & 1.08 & 6 & 2.33 & 126 & 0.69 & 9 & 1.22 & $0.4(p>.05)$ \\
\hline & & D25.9 & 0 & 0.00 & 0 & 0.00 & 41 & 0.54 & 1 & 0.39 & 41 & 0.22 & 1 & 0.14 & $0.0(p=1.0)$ \\
\hline & & C50 & 1 & 0.01 & 0 & 0.00 & 14 & 0.18 & 0 & 0.00 & 15 & 0.08 & 0 & 0.00 & NA \\
\hline & & C16 & 8 & 0.07 & 0 & 0.00 & 6 & 0.08 & 0 & 0.00 & 14 & 0.08 & 0 & 0.00 & NA \\
\hline \multirow{4}{*}{ (D50-D89) } & $\begin{array}{l}\text { Diseases of } \\
\text { forming orga } \\
\text { involving the }\end{array}$ & $\begin{array}{l}\text { blood \& blood } \\
\text { certain disorders } \\
\text { mune mechanism }\end{array}$ & 371 & 3.46 & 14 & 2.90 & 380 & 5.02 & 12 & 4.65 & 751 & 4.10 & 26 & 3.51 & $1.0(p>.05)$ \\
\hline & & D64.9 & 221 & 2.06 & 7 & 1.45 & 305 & 4.03 & 11 & 4.26 & 526 & 2.87 & 18 & 2.43 & $0.2(p>.05)$ \\
\hline & & D56.9 & 93 & 0.87 & 1 & 0.21 & 45 & 0.59 & 0 & 0.00 & 138 & 0.75 & 1 & 0.14 & $0.0(p=1.0)$ \\
\hline & & D61.9 & 18 & 0.17 & 0 & 0.00 & 16 & 0.21 & 0 & 0.00 & 34 & 0.19 & 0 & 0.00 & $1.2(p>.05)$ \\
\hline & $\begin{array}{l}\text { Endocrine, } n \\
\text { bolic disease }\end{array}$ & itional and meta- & 244 & 2.27 & 22 & 4.56 & 226 & 2.98 & 22 & 8.53 & 470 & 2.57 & 44 & 5.95 & $0.5(p>.05)$ \\
\hline (E00-E99) & & E10-E14 & 15 & 0.14 & 20 & 4.15 & 126 & 1.66 & 16 & 6.20 & 279 & 1.52 & 36 & 4.86 & $0.2(p>.05)$ \\
\hline & & E88.9 & 46 & 0.43 & 0 & 0.00 & 49 & 0.65 & 0 & 0.00 & 95 & 0.52 & 0 & 0.00 & $0.1(p>.05)$ \\
\hline & & E46 & 36 & 0.34 & 2 & 0.41 & 29 & 0.38 & 2 & 0.78 & 65 & 0.36 & 4 & 0.54 & $0.1(p>.05)$ \\
\hline & Mental and $\mathrm{t}$ & lavioural disorders & 37 & 0.34 & 1 & 0.21 & 15 & 0.20 & 1 & 0.39 & 52 & 0.28 & 2 & 0.27 & $0.1(p>.05)$ \\
\hline (E00-509) & & F10.2 & 24 & 0.22 & 0 & 0.00 & 0 & 0.00 & 0 & 0.00 & 24 & 0.13 & 0 & 0.00 & NA \\
\hline ) & & F29 & 8 & 0.07 & 1 & 0.21 & 7 & 0.09 & 1 & 0.39 & 15 & 0.08 & 2 & 0.27 & $0.0(p=1.0)$ \\
\hline & & F41.1 & 5 & 0.05 & 0 & 0.00 & 8 & 0.11 & 0 & 0.00 & 13 & 0.07 & 0 & 0.00 & $0.1(p>.05)$ \\
\hline & Diseases of & nervous system & 105 & 0.98 & 17 & 3.53 & 81 & 1.07 & 8 & 3.10 & 186 & 1.02 & 25 & 3.38 & $0.1(p>.05)$ \\
\hline Icongoo & & G81.9 & 31 & 0.29 & 3 & 0.62 & 19 & 0.25 & 2 & 0.78 & 5 & 0.03 & 5 & 0.68 & $0.1(p>.05)$ \\
\hline (נ00000 & & G03.9 & 26 & 0.24 & 3 & 0.62 & 21 & 0.28 & 0 & 0.00 & 47 & 0.26 & 3 & 0.41 & $0.1(p>.05)$ \\
\hline & & G04.9 & 8 & 0.07 & 5 & 1.04 & 13 & 0.17 & 5 & 1.94 & 21 & 0.11 & 10 & 1.35 & $0.0(p=1.0)$ \\
\hline & Diseases of & eye and adnexa & 643 & 5.99 & 0 & 0.00 & 823 & 10.9 & 0 & 0.00 & 1466 & 8.01 & 0 & 0.00 & $3.3(p<.05)^{\star}$ \\
\hline & & H25.2 & 415 & 3.87 & 0 & 0.00 & 583 & 7.70 & 0 & 0.00 & 998 & 5.45 & 0 & 0.00 & $2.5(p<.05)^{\star}$ \\
\hline (1) & & H26.9 & 188 & 1.75 & 0 & 0.00 & 237 & 3.13 & 0 & 0.00 & 425 & 2.32 & 0 & 0.00 & $0.8(p>.05)$ \\
\hline & & $\mathrm{H} 44.8$ & 40 & 0.37 & 0 & 0.00 & 3 & 0.04 & 0 & 0.00 & 43 & 0.23 & 0 & 0.00 & $0.1(p>.05)$ \\
\hline & $\begin{array}{l}\text { Diseases of } \\
\text { process }\end{array}$ & ear and mastoid & 85 & 0.79 & 0 & 0.00 & 107 & 1.41 & 0 & 0.00 & 192 & 1.05 & 0 & 0.00 & $0.4(p>.05)$ \\
\hline (H60-H95) & & $\mathrm{H} 66.3$ & 67 & 0.62 & 0 & 0.00 & 90 & 1.19 & 0 & 0.00 & 157 & 0.86 & 0 & 0.00 & $0.4(p>.05)$ \\
\hline & & H81 & 18 & 0.17 & 0 & 0.00 & 17 & 0.22 & 0 & 0.00 & 35 & 0.19 & 0 & 0.00 & $0.0(p=1.0)$ \\
\hline & $\begin{array}{l}\text { Diseases of } \\
\text { system }\end{array}$ & circulatory & 426 & 3.97 & 49 & 10.17 & 303 & 4.00 & 26 & 10.08 & 729 & 3.98 & 75 & 10.14 & $0.0(p=1.0)$ \\
\hline$(100-199)$ & & 110 & 162 & 1.51 & 14 & 2.90 & 117 & 1.54 & 9 & 3.49 & 279 & 1.52 & 23 & 3.11 & $0.2(p>.05)$ \\
\hline & & 164 & 163 & 1.52 & 26 & 5.39 & 88 & 1.16 & 10 & 3.88 & 251 & 1.37 & 36 & 4.86 & $0.0(p=1)$ \\
\hline & & 109.9 & 14 & 0.13 & 3 & 0.62 & 34 & 0.45 & 1 & 0.39 & 48 & 0.26 & 4 & 0.54 & $0.2(p>.05)$ \\
\hline & $\begin{array}{l}\text { Diseases of } \\
\text { system }\end{array}$ & respiratory & 1131 & 10.5 & 78 & 16.2 & 541 & 7.1 & 32 & 12.4 & 1672 & 9.1 & 110 & 14.9 & $2.2(p<.05)^{\star}$ \\
\hline (J00-J99) & & J44.9 & 676 & 6.30 & 66 & 13.69 & 301 & 3.97 & 24 & 9.30 & 977 & 5.34 & 90 & 12.16 & $0.1(p>.05)$ \\
\hline & & $\mathrm{J} 34.2$ & 90 & 0.84 & 0 & 0.00 & 24 & 0.32 & 0 & 0.00 & 114 & 0.62 & 0 & 0.00 & $0.3(p>.05)$ \\
\hline & & J18.9 & 61 & 0.57 & 1 & 0.21 & 29 & 0.38 & 1 & 0.39 & 90 & 0.49 & 2 & 0.27 & $1.4(p>.05)$ \\
\hline & Diseases of & digestive system & 618 & 5.76 & 17 & 3.53 & 305 & 4.03 & 17 & 6.59 & 923 & 5.04 & 34 & 4.59 & $1.2(p>.05)$ \\
\hline$(K 00 \mathrm{k} 02)$ & & K75.9 & 73 & 0.68 & 0 & 0.00 & 39 & 0.51 & 3 & 1.16 & 112 & 0.61 & 3 & 0.41 & $0.1(p>.05)$ \\
\hline (לת) & & K40.9 & 100 & 0.93 & 0 & 0.00 & 9 & 0.12 & 0 & 0.00 & 109 & 0.60 & 0 & 0.00 & $0.3(p>.05)$ \\
\hline & & K29.7 & 53 & 0.49 & 0 & 0.00 & 54 & 0.71 & 1 & 0.39 & 107 & 0.58 & 1 & 0.14 & $0.1(p>.05)$ \\
\hline
\end{tabular}


Citation: Tyagi BB, Aswal RS, Haroon AS (2015) Morbidity Patterns Amongst Hospitalized Patients in a Secondary Care Hospital of Dehradun, Uttarakhand State, India. J intern Med Prim Healthcare 1: 003.

- Page 7 of $10 \bullet$

\begin{tabular}{|c|c|c|c|c|c|c|c|c|c|c|c|c|c|c|}
\hline \multirow{4}{*}{ (L00- L99) } & $\begin{array}{l}\text { Diseases of the skin and subcuta- } \\
\text { neous tissue }\end{array}$ & \multirow{2}{*}{$\begin{array}{l}63 \\
43\end{array}$} & \multirow{2}{*}{$\begin{array}{l}0.59 \\
0.40\end{array}$} & \multirow[b]{2}{*}{1} & \multirow{2}{*}{$\begin{array}{l}0.41 \\
0.21\end{array}$} & \multirow{2}{*}{$\begin{array}{l}39 \\
24\end{array}$} & \multirow{2}{*}{$\begin{array}{l}0.51 \\
0.32\end{array}$} & \multirow{2}{*}{$\begin{array}{l}1 \\
0\end{array}$} & \multirow{2}{*}{$\begin{array}{l}0.39 \\
0.00\end{array}$} & \multirow{2}{*}{$\begin{array}{l}102 \\
67\end{array}$} & \multirow{2}{*}{$\begin{array}{l}0.56 \\
0.37\end{array}$} & \multirow{2}{*}{3} & \multirow{2}{*}{$\begin{array}{l}0.41 \\
0.14\end{array}$} & \multirow{2}{*}{$\begin{array}{l}0.1(p>.05) \\
0.1(p>.05)\end{array}$} \\
\hline & L02.9 & & & & & & & & & & & & & \\
\hline & L03.9 & 24 & 0.22 & 1 & 0.21 & 14 & 0.18 & 1 & 0.39 & 38 & 0.21 & 2 & 0.27 & $0.0(p=1.0)$ \\
\hline & L08.9 & 0 & 0.00 & 0 & 0.00 & 10 & 0.13 & 0 & 0.00 & 10 & 0.05 & 0 & 0.00 & NA \\
\hline \multirow{2}{*}{ (M00-M99) } & $\begin{array}{l}\text { Diseases of the musculoskeletal } \\
\text { system and connective tissue }\end{array}$ & 13 & 0.12 & 0 & 0.00 & 20 & 0.26 & 0 & 0.00 & 33 & 0.18 & 0 & 0.00 & $0.1(p>.05)$ \\
\hline & M54.5 & 13 & 0.12 & 0 & 0.00 & 20 & 0.26 & 0 & 0.00 & 33 & 0.18 & 0 & 0.00 & $0.1(p>.05)$ \\
\hline \multirow{4}{*}{ (N00-N99) } & $\begin{array}{l}\text { Diseases of the genito- urinary } \\
\text { system }\end{array}$ & 256 & 2.38 & 13 & 2.70 & 264 & 3.49 & 7 & 2.71 & 520 & 2.84 & 20 & 2.70 & $0.7(p>.05)$ \\
\hline & $\mathrm{N} 20$ & 46 & 0.43 & 0 & 0.00 & 44 & 0.58 & 0 & 0.00 & 90 & 0.49 & 0 & 0.00 & $0.0(p=1.0$ \\
\hline & N18 & 44 & 0.41 & 7 & 1.45 & 27 & 0.36 & 4 & 1.55 & 71 & 0.39 & 11 & 1.49 & $0.1(p>.05)$ \\
\hline & $\mathrm{N} 43.3$ & 60 & 0.56 & 0 & 0.00 & 0 & 0.00 & 0 & 0.00 & 60 & 0.33 & 0 & 0.00 & NA \\
\hline \multirow{3}{*}{ (Q99-Q99) } & $\begin{array}{l}\text { Congenital malformation } \\
\text { deformations \& chromosomal } \\
\text { abnormality }\end{array}$ & 2 & 0.02 & 0 & 0.00 & 12 & 0.16 & 0 & 0.00 & 14 & 0.08 & 0 & 0.00 & $0.1(p>.05)$ \\
\hline & Q50.1 & 0 & 0.00 & 0 & 0.00 & 12 & 0.16 & 0 & 0.00 & 12 & 0.07 & 0 & 0.00 & NA \\
\hline & Q64.7 & 2 & 0.02 & 0 & 0.00 & 0 & 0.00 & 0 & 0.00 & 2 & 0.01 & 0 & 0.00 & NA \\
\hline \multirow{4}{*}{ (R00-R99) } & $\begin{array}{l}\text { Symptoms, signs \& abnormal } \\
\text { clinical \& laboratory findings, not } \\
\text { elsewhere class. }\end{array}$ & 3070 & 28.60 & 138 & 28.63 & 2311 & 30.51 & 77 & 29.84 & 5381 & 29.39 & 215 & 29.05 & $1.5(p>.05)$ \\
\hline & R50.9 & 2388 & 22.24 & 102 & 21.16 & 1791 & 23.65 & 63 & 24.42 & 4179 & 22.82 & 165 & 22.30 & $0.3(p>.05)$ \\
\hline & R10.4 & 224 & 2.09 & 7 & 1.45 & 190 & 2.51 & 2 & 0.78 & 414 & 2.26 & 9 & 1.22 & $1.1(p>.05)$ \\
\hline & R56.8 & 111 & 1.03 & 2 & 0.41 & 80 & 1.06 & 0 & 0.00 & 191 & 1.04 & 2 & 0.27 & $0.1(p>.05)$ \\
\hline \multirow{4}{*}{ (S00-T98) } & $\begin{array}{l}\text { Injury, poisoning and certain other } \\
\text { consequences of external causes }\end{array}$ & 1359 & 12.7 & 38 & 7.9 & 610 & 8.0 & 13 & 5.0 & 1969 & 10.8 & 51 & 6.9 & $3.0(p<.05)^{\star}$ \\
\hline & T14.2 & 492 & 4.58 & 3 & 0.62 & 186 & 2.46 & 2 & 0.78 & 678 & 3.70 & 5 & 0.68 & $1.2(p>.05)$ \\
\hline & T65.9 & 249 & 2.32 & 12 & 2.49 & 167 & 2.20 & 5 & 1.94 & 416 & 2.27 & 17 & 2.30 & $0.8(p>.05)$ \\
\hline & T14.9 & 286 & 2.66 & 1 & 0.21 & 105 & 1.39 & 1 & 0.39 & 391 & 2.14 & 2 & 0.27 & $0.1(p>.05)$ \\
\hline \multirow{4}{*}{ (V01-Y98) } & $\begin{array}{l}\text { External causes of morbidity and } \\
\text { mortality }\end{array}$ & 469 & 4.4 & 28 & 5.8 & 123 & 1.6 & 2 & 0.8 & 592 & 3.2 & 30 & 4.0 & $1.4(p>.05)$ \\
\hline & $\times 59$ & 259 & 2.41 & 15 & 3.11 & 46 & 0.61 & 0 & 0.00 & 275 & 1.50 & 15 & 2.03 & $0.6(p>.05)$ \\
\hline & V99 & 200 & 1.86 & 13 & 2.70 & 55 & 0.73 & 0 & 0.00 & 255 & 1.39 & 13 & 1.76 & $0.0(p=1.0)$ \\
\hline & W17 & 24 & 0.22 & 0 & 0.00 & 18 & 0.24 & 0 & 0.00 & 42 & 0.23 & 0 & 0.00 & $0.7(p>.05)$ \\
\hline \multicolumn{2}{|c|}{ TOTAL of all disease category (A00-Y98) } & 10735 & 100.0 & 482 & 100.0 & 7574 & 100.0 & 258 & 100.0 & 18309 & 100.0 & 740 & 100.0 & $22.9(p<.05)$ \\
\hline
\end{tabular}

Table 4: Distribution of morbidity and mortality pattern of diseases.

DSSACLF NEC**

Certain infectious and parasitic diseases

Injury, poisioning and certain other conseq.

Diseases of the respiratory system

Diseases of eye and adnexa

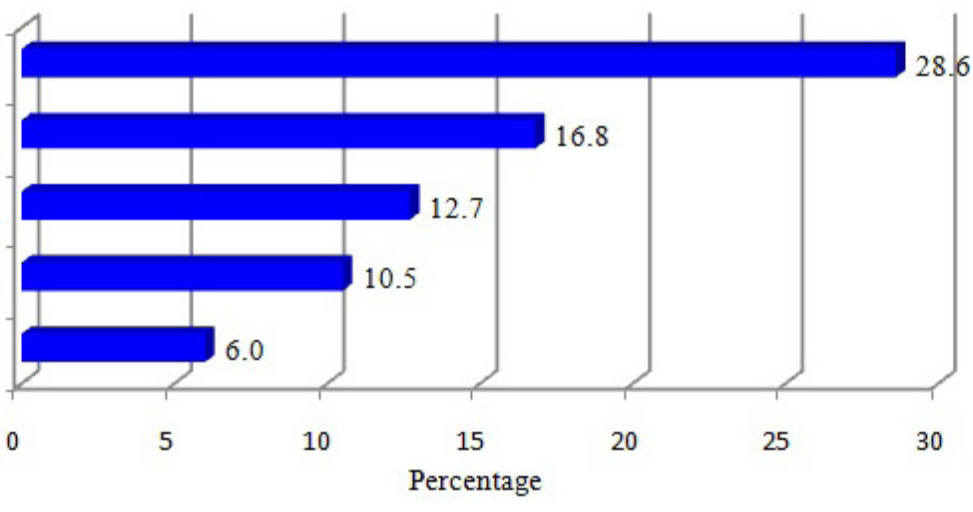

Figure 4: Leading causes of morbidity in males. 
abnormal clinical and laboratory findings, not elsewhere classified [ICD.10:R00-R99, $\mathrm{n}=3070$ (28.6\%)] followed by infectious parasitic disease [ICD.10:A00-B99, $\mathrm{n}=1799(16.8 \%)$ ], injury, poisoning and certain other consequences of external cause [IDC.10:S00-T98, $\mathrm{n}=1359(12.7 \%)$ ], diseases of respiratory system [ICD.10:J00-J99, $\mathrm{n}=1131(10.5 \%)$ ] and diseases of eye and adnexa [ICD.10:H00-H59, $\mathrm{n}=643(6.0 \%)]$ (Figure 4$)$.

In females the leading causes of morbidity were symptoms, signs and abnormal clinical and laboratory findings, not elsewhere classified cured patients $[\mathrm{n}=10271(56.1 \%)]$ in both sexes was much higher as compared to the other conditions at discharge. The proportions of referrals in the age group of 35-64 years (46.2\%) were higher as compared to other age groups. A significant proportion of patients left the health facility against medical advice (17.0\%). The probable reason would be either non-satisfaction with the medical care available or inability to continue the treatment at the facility. The referral (3.4\%) rate was substantially low as compared to the other conditions of discharge. This may probably due to the fact that our

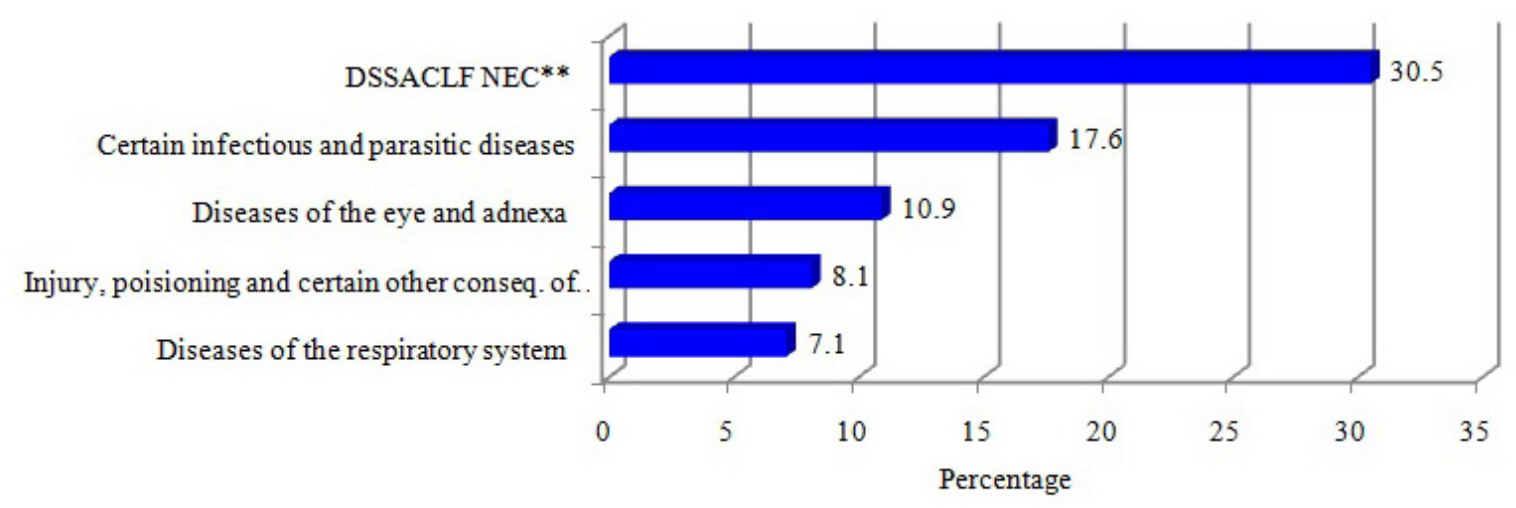

Figure 5: Leading causes of morbidity in females.

DSSACLF NEC ** - Diseases of symptoms, signs and abnormal clinical and laboratory findings not elsewhere classified

$[\mathrm{n}=2311(30.5 \%)]$ followed by infectious parasitic disease $[\mathrm{n}=1332$ $(17.6 \%)]$, diseases of eye and adnexa $[n=823(10.9 \%)]$, injury, poisoning and certain other consequences of external cause $[n=610$ $(8.1 \%)]$ and diseases of respiratory system $(n=541 \quad(7.1 \%)]$ (Figure 5). Statistically significant differences were seen among males and females for morbidity disease category of eye and adnexa $3.3(\mathrm{p}<0.05)$, diseases of the respiratory system $2.2(\mathrm{p}<0.05)$ and morbidity disease category of injury, poisoning and certain other consequences of external causes $3.0(\mathrm{p}<0.05)$.

In males and females, leading causes of mortality were symptoms, signs and abnormal clinical and laboratory findings with $28.6 \%$ and $29.8 \%$ respectively, followed by diseases of respiratory system $(16.2 \%$ and $12.4 \%$ ), infectious parasitic disease (12.9\% and $13.2 \%)$, diseases of circulatory system (10.2\% and 10.1\%) respectively (Figures 6 and 7).

\section{Discussion}

This study only analyzed hospital admissions, which did not include patients seen in the emergency and Outpatients (OPD) Departments per se. Nevertheless, similar results would be expected if OPD patients were to be included, since most of them present there for post-hospitalization follow-up, although some patients with more acute problems are referred directly to the OPD. Despite obvious limitations associated with the validity of hospital records (eg: omissions, inaccurate recording of diagnosis at the time of admission, etc.), the examination of such data has still yielded a reasonably representative picture of the prevailing ills in the community served by Doon Hospital. A significant proportion of facility follows the standards/guidelines laid down in Indian Public Health Standards (IPHS) [16]. Results of the District Level Household Survey - III 2007-08 (DLHS - III) shows majority of health facilities at secondary care does not conform to IPHS standards. The overall mortality rate of 4.0 per thousand admissions in our study is comparable with the mortality rate of other secondary care centers [17]. Some limitations are being noted in this study. Being a retrospective study, extraction of final diagnoses from patient's folders who presented with multiple complaints/morbidities could alter the actual prevalence of the disease entities so recorded. Secondly we relied on the clinical soundness of the attending physician in patients' diagnoses which may vary from individual to individual based on experience, qualifications and other factors.

\section{Conclusion}

The present study only highlights the status of different types of morbidities, mortalities and bed occupancy rate in a secondary health care hospital. This is the first study amongst admitted patients of all causes of morbidity and mortality with all ages to highlight the morbidity and mortality pattern of hospitalized patients in all ages and the case fatality rate.

\section{Acknowledgements}

Author will like to acknowledge Dr. BC Pathak (Ex DG), Shri Piyush Singh (Addl Health Secretary \& Mission Director), Director General of Medical Health Services \& Medical Superintendent of 
Citation: Tyagi BB, Aswal RS, Haroon AS (2015) Morbidity Patterns Amongst Hospitalized Patients in a Secondary Care Hospital of Dehradun, Uttarakhand State, India. Jintern Med Prim Healthcare 1: 003.

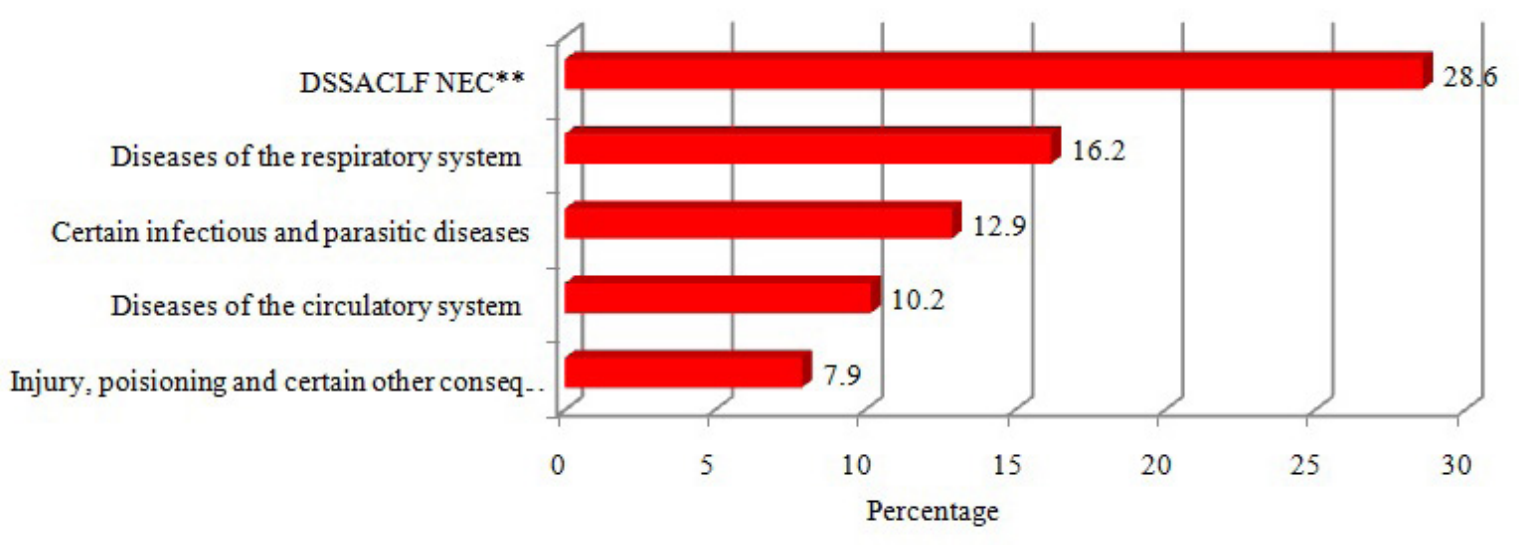

Figure 6: Leading causes of mortality in males.

DSSACLF NEC ${ }^{* *}$ - Diseases of symptoms, signs and abnormal clinical and laboratory findings not elsewhere classified

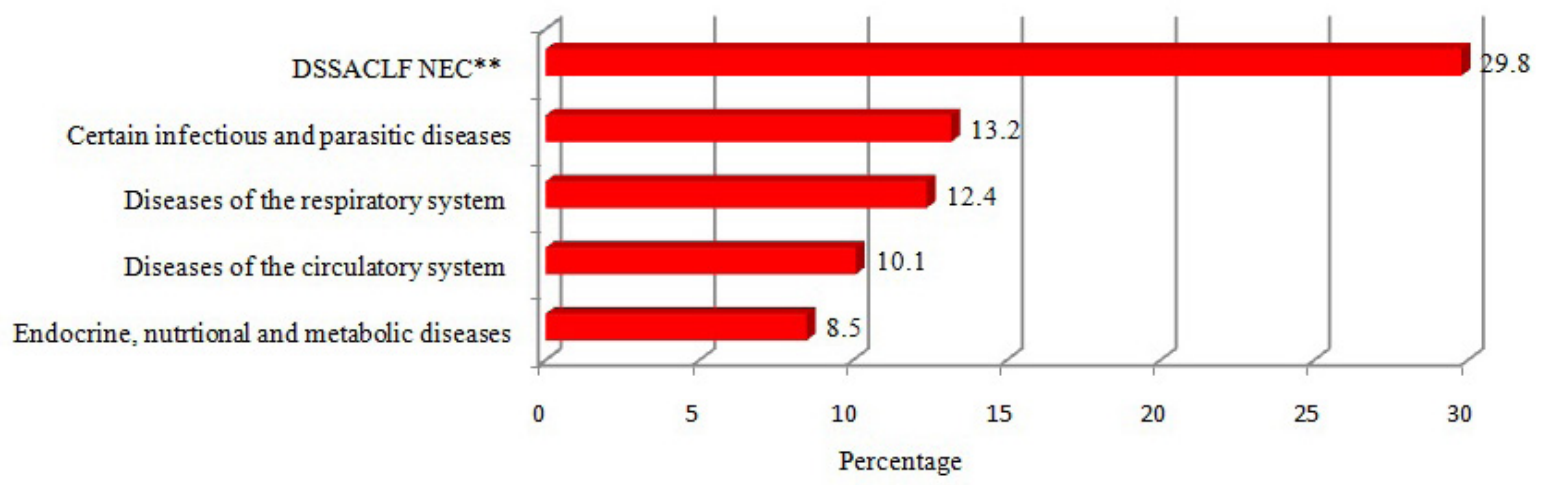

Figure 7: Leading causes of mortality in females.

DSSACLF NEC**- Diseases of symptoms, signs and abnormal clinical and laboratory findings not elsewhere classified

Doon hospital, Government of Uttarakhand who given permission to collect the valuable data of this research work.

A very special expression of appreciation is extended to Shri VK Negi (Statistical Officer) \& to the medical records staff for their assistance in retrieving the relevant case notes.

\section{References}

1. Annual Report 2010-11 (2011) Ministry of Health and Family Welfare. Department of Health and Family Welfare.

2. International Institute for Population Sciences (IIPS) (1999) National Family Health Survey (NFHS-2) 1998-99. International Institute for Population Sciences (IIPS), Mumbai, India.

3. Ministry of Home affairs (2008) SRS Bulletin. Sample Registration System, Registrar General, Ministry of Home affairs, Government of India, New Delhi. Volume 43: 1.

4. Ministry of Health and Family Welfare (2011) Family Welfare Statistics in India. Statistics Division, Ministry of Health and Family Welfare, Government of India, India.

5. Duraiswamy P (1998) Morbidity in Tamil Nadu- Level, Differentials and Determinants. Economic and Political Weekly XXXII: 982-990.
6. Kirit S Parikh (1999) India Development Report 1999-2000. Indira Gandh Development Research Institute. Oxford University Press, New Delhi, India. Pg no: 51.

7. Bhalwar R (2004) Prospective Study on the Morbidity Profile of Recruits over one year in three large Regimental Training Centre. MJAFI 60:113-116.

8. National Health Portal (2015) National Health Policy 2002. Department of Health and Family Welfare. Ministry of Health and Family Welfare, Government of India, New Delhi, India.

9. Ministry of Health and Family Welfare (2006) National Rural Health Mission (2005-2012). Mission Document. NRHM, Department of Health and Family Welfare, Ministry of Health and Family Welfare, Government of India, New Delhi, India.

10. Davies RL, Macaulay HMC (1966) Hospital Planning and Administration, WHO Monograph series No. 54. World Health Organization, Geneva, Switzerland.

11. Davies RL, Macaulay HMC (1995) Hospital planning and administration, WHO monograph series. No. 54. World Health Organization, Geneva. Jaypee Brothers, India. Pg no: 6-35

12. Uttarakhand Government Portal (2015) Home. Uttarakhand Government Portal, Uttarakhand, India. 
Citation: Tyagi BB, Aswal RS, Haroon AS (2015) Morbidity Patterns Amongst Hospitalized Patients in a Secondary Care Hospital of Dehradun, Uttarakhand State, India. J intern Med Prim Healthcare 1: 003.

- Page 10 of $10 \bullet$

13. Census of India (2011) Uttarakhand Population Census data 2011. Census 2011, Census of India, India.

14. World Health Organization Geneva (2010) International Statistical Classification of Diseases and Related Health Problems (10 ${ }^{\text {th }}$ Revision) (Vol 2). World Health Organization, Geneva, Switzerland.

15. World Whether Online (2015) Dehradun Weather. Uttarakhand, India, Country list, World Whether Online.
16. National Rural Health Mission (2013) Indian Public Health Standards. Directorate General of Health Services, National Rural Health Mission, Ministry of Health \&Family Welfare, Government of India, India.

17. Directorate General of Health Services (2006) Indian Public Health Standards (IPHS) for primary health centers, Guidelines. Directorate General of Health Services, Ministry of Health \& Family Welfare, Government of India, New Delhi, India. 